\title{
Theoretical Design of Energy Generating Gymnasium Pull-down Machine for Green, Renewable and Sustainable Energy Production
}

\author{
M. Musharraf, ${ }^{1, *}$ Ifrah Saleem, ${ }^{2}$ and Dr. Farhat Iqbal ${ }^{1}$ \\ ${ }^{I}$ Department of Electrical Engineering, University College of Engineering and Technology (UCET), \\ University of Sargodha (UOS), Sargodha, Pakistan 40100 \\ ${ }^{2}$ University of Lahore (UOL), Lahore, Pakistan 54000
}

Received November 16, 2018; Accepted December 23, 2018; Published December 24, 2018

\begin{abstract}
New technologies are being invented and energy demand is increasing. Growth of population has always been and will remain one of the major causes of energy demand. Science is therefore looking for new major and minor energy resources to keep world in progress. The main focus of energy engineering and technology in the field of energy generation is to harvest energy by any mean from any source. A theoretical research is introduced in this paper which will contribute its reasonable share in the field of renewable and green energy sector. This energy generating system is named as energy generating gymnasium system (EGGS). The core idea behind this energy harvesting system is that, the human being is also a source of renewable energy and it is possible to harness electrical energy from people by the use of EGGS. Human energy is wasted when excessive calories of body are burnt during exercise in gymnasiums to achieve the desired fitness. EGGS will provide an opportunity to return expended energy in the form of electrical energy from gymnasium equipment and cardiovascular machines. This electrical energy will be cheap and also green since it will not emit any carbon dioxide $\left(\mathrm{CO}_{2}\right)$ gas during the process. This system can increase the potential of renewable energy area and the electrical energy generated from EGGS can be sold back to the national utility via micro grids (MG). The proposed system will be very beneficial for such countries that are facing energy crises as well as the third world countries. Authors have discussed a gymnasium machine named as "Pull-down machine" and proposed a theoretical modification to make it as an energy generating gymnasium machine (EGGM) in the presented research.
\end{abstract}

Keywords: EGGS; EGGM; Gymnasium; Renewable energy; Cable and pulley; Pull-down machine; Micro grid

\section{Introduction}

The world around us is changing significantly and the use of technology has become one of the major drivers for economic and social development. Rapid advancement in engineering and Information Technology (IT) all over the world has transformed not only the way people think, but also the way people act. Most of the technologies require electricity for their proper operation and consequently the share of electricity is increasing 
rapidly than total primary energy generation/supply. World total final consumption (TFC) by fuel was 9,384 metric tons oil equivalents (MTOE) in 2015 and by 2040 it will be 10,706 MTOE [1]. World net electricity generation increases by $45 \%$, rising from 23.4 trillion kilowatt hours (kWh) in 2015 to 34.0 trillion $\mathrm{kWh}$ in 2040 [2]. World net electricity generation increases $69 \%$ by 2040, from 21.6 trillion kilowatt hours (kWh) in 2012 to 25.8 trillion $\mathrm{kWh}$ in 2020 and 36.5 trillion $\mathrm{kWh}$ in 2040.

The great outdoor (TGO) gymnasium company in United Kingdom (UK) has been generating electrical energy through playing and exercise by using energy generating gymnasium equipment (EGGE) and has installed green energy generating fitness machines at Sir George Monoux College, Trafalgar Square, and the Green Heart in Hull city [3, 4]. TGO Company has launched outdoor gymnasium machines, which offer a cardiac workout and generate electricity, and has upgraded the Cross Trainer, Recumbent Bike, Spinning Bike, and Hand Bike to green energy gymnasium equipment that generates on average 50100 watts each depending on the fitness of the user [3]. A generator is connected to these machines in such a way as the circular rotation of the front wheel rotates the coils of wires inside the generator between the poles of the magnets [5]. These machines generate electricity from their rotating parts, which lead the authors towards an idea of extracting energy from gymnasium machines that do not exhibit rotating motion but have linear moving parts.

This research presents a possibility of electricity generation from linear motion of Pull-down machine whenever the user applies force on it by expending energy. Solar energy is converted into chemical energy by plants and this chemical energy is stored in human body by consuming plants as shown in Fig 1.The conversion of this stored chemical energy in human body into the electrical energy is the focus of this research. Solar radiation is an ultimate source for generating renewable, sustainable, and environmentally friendly energies. There are various ways of harvesting and using solar energy for fulfilling the energy needs of the modern society. Solar energy arrives at planet earth through radiation and photosynthesis is the process to bring and spreads solar energy into the living system through food chain and food web. Plants and algae like cyanobacteria convert light energy into chemical energy through photosynthesis naturally, which can be later released and used as an energy source by these organisms to fuel their activities. The converted chemical energy is stored in the form of carbohydrates such as sugars, which are synthesized from $\mathrm{CO}_{2}$ and water. The process of photosynthesis not only generates fuel but it also releases

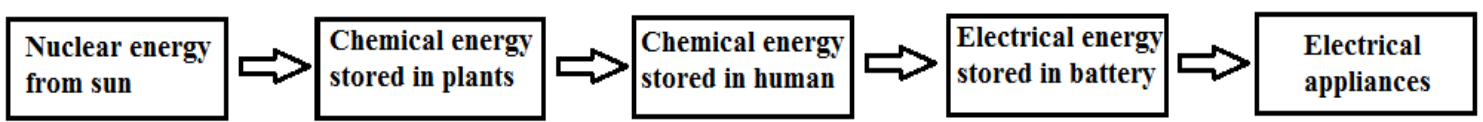

Figure 1. Human body converts solar nuclear energy into electrical energy

oxygen as a by-product. The benefits of mimicking the process of photosynthesis can benefit living organisms on earth twofold. There are producers and energy converter in this natural cycle in which producers make food (glucose) by using water and sun light. Human consumes this food and chemical energy of this food, which is converted into mechanical energy whenever some useful work is done, and this mechanical energy can be converted into electrical energy with the help of the energy generating gymnasium machines (EGGM). Energy generating gymnasiums will be the places in future where 
electrical energy can be harvested from renewable (non-conventional) energy resources (human beings).

\section{Classification of Energy Resources}

Generally, the energy resources have been classified as "conventional and renewable energy resources (non-conventional)".

\subsection{Conventional Energy Resources}

These energy resources includes fossil oil, natural gas, coal and some metals like uranium and plutonium. Conventional energy resources remain fixed in nature. According to world energy council [6], Fossil oil remained the world's leading fuel, accounting for $32.9 \%$ of global energy consumption and roughly $63 \%$ of oil consumption comes from the transport sector. Natural gas is the second largest energy source in power generation, representing $22 \%$ of generated power globally. Coal still provides around $40 \%$ of the world's electricity and Asia presents the biggest market for coal and currently accounts for $66 \%$ of global coal consumption. Kazakhstan is the world's leading producer of uranium. Global uranium production increased by 40\% between 2004 and 2013.

\subsection{Renewable Energy Resources}

These are resources of energy which regenerate themselves naturally after specific time interval and will never come to an end. Hydro power is the leading renewable source for electricity generation globally, supplying $71 \%$ of all renewable electricity at the end of 2015 and undeveloped potential is approximately 10,000 Tara watt hour per year (TWh/y) worldwide [6]. Global wind power generation capacity reached 432 Giga watt (GW) in 2015, around 7\% of total global power generation capacity (420 GW onshore, $12 \mathrm{GW}$ offshore). Global installed capacity for solar-powered electricity has seen an exponential growth, reaching around $227 \mathrm{GW}$ at the end of 2015, producing $1 \%$ of all electricity used globally. Global output of Geothermal is estimated to be $75 \mathrm{TWh}$ for heat and $75 \mathrm{TWh}$ for power, but is concentrated on geologic plate boundaries [6].

Adenosine Triphosphate (ATP) is a source of energy which is stored in human muscles and available for muscles movements. Hence any movement that is last longer than a few seconds more ATP is produced in human body. Presented research suggests that ATP possessed by human body is a source of renewable energy and may be converted into electrical energy. ATP is regenerated by addition of a phosphate group to adenosine di-phosphate (ADP) in human body [7, 8]. Energy is required when the
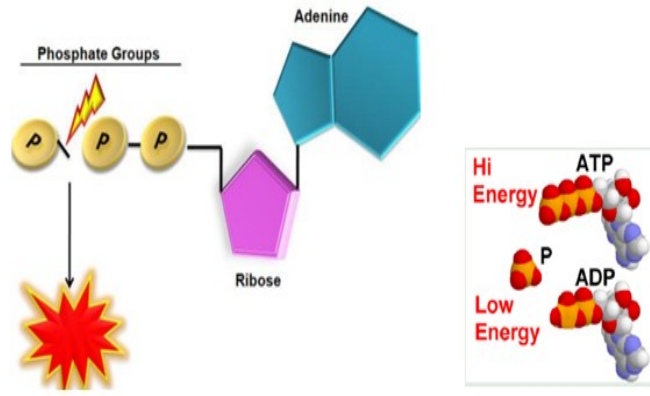

Figure 2. Energy released for cell metabolism phosphate bond is formed with ADP which results in ATP and this ATP stores in human body. When phosphate group is removed the ATP breaks apart and releases its energy and becomes ADP, the ATP - ADP cycle is a revolving door [9]. ADP utilizes energy and gains an extra " $p$ " and is recharged back to ATP [10]. Cell's energy currency-ATP, is a bigger molecule consisting of five smaller 
units i.e., adenine, ribose, and three phosphate groups which are shown in Fig 2. Catabolic reactions in human cell provide energy to phosphorylate ADP into ATP. In a catabolic reaction large molecules are broken down into small ones and release energy. ATP cycle is synchronized with transfer of energy from catabolic to anabolic pathways [11]. ATP contains more energy than ADP because it has more bonds. Energy is necessary to produce
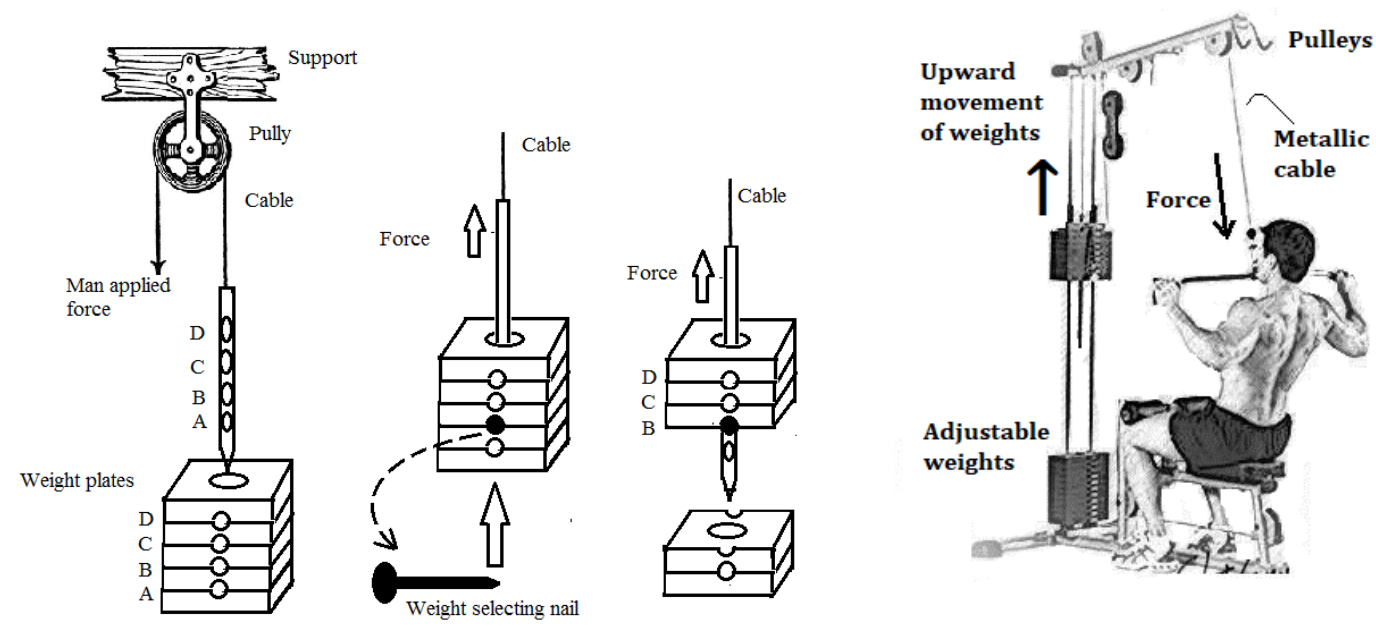

Figure 3. Working principle of pull-down machine

physical movement, maintain body temperature and metabolic activities. Human body has three systems to produce ATP [12].

1) ATP-PC (phosphocreatine System) - High power short duration

2) Glycolytic system - Moderate power/ moderate duration

3) Oxidative system - Low power/ long duration

First two are anaerobic systems; it means oxygen is not required to produce ATP. Last one oxidative system is aerobic which requires oxygen for ATP production. In ATP-PC system, movements last about 5 to 15 seconds, such as power movements, jumps and throwing ball, which can be done by the ATP stored in the muscles [13]. ATP-PC system produces energy very quickly but not over a long duration. Next energy domain shifted to glycolytic system, in this system ATP is generated by a process called as Glycolysis in which rapid break down of carbohydrates into glucose occurs. The generated amount of Glucose constantly circulates in the human blood. Glycolytic system produces energy quickly however again not for a long duration. Oxidative system involves the use of oxygen, fats, carbohydrates and sometimes proteins for resynthesizing ATP. This system produces far more ATP than either of the energy systems however it produces the ATP much more slowly.

\section{Proposed Work}

Energy demand is ascending day by day and humankind is exploring new ideas and resources from where they can extract any form of energy. Biomass energy is created by the combustion or biochemical conversion of an organic matter to be used for fuel. Some of organic materials used as a source for biomass energy include wood, sawdust, grasses, corn, sugarcane, and farm waste (cow manure). The processes for converting biomass into 
energy are numerous, which are: combustion process, co-firing process, thermal conversion, gasification, pyrolysis, chemical conversion, biomass oil, fermentation process, and torrefaction. This research will discuss a method in order to harvest energy from biomass. In the proposed method, human will act as a device or machine for converting biomass energy into electrical energy. The device which converts one form of energy into another form of energy is called a transducer, so human could be considered as a transducer.

A person gains calories from food and performs activities of daily life, however excess of calories, unbalanced diet and extra fats make him unhealthy. Gymnasiums are the place where different types of machines are available to perform exercises like running, weight lifting, and muscles building in order to burn the excess of calories and fats. There are specified machines to perform exercise of a particular portion of human body e.g., cable preacher curl, close grip bar curl, barbell curl, and dumbbells lifting are some exercises for biceps. Chest exercises include barbell bench press, flat bench dumbbell press, low inclined barbell bench press, seated machine bench press, etc. As the demand of energy is increasing day by day, it is essential to invent new ways and techniques to produce energy within a small area which may be a gymnasium or home. Most of cardiovascular machines in gymnasiums are made of cables and pulleys and these machines are the main focus of this research, because these machines can contribute in harvesting energy. During exercise, persons apply force on machines and expend energy to do some work. This expenditure of energy may be converted in other form of energy e.g., in the form of electrical energy. It is possible to construct gymnasium machines that can convert and store expended energy by the users into electrical energy throughout the exercise $[14,15]$.

\subsection{Working Principle of "Pull-down" Machine}

Pull-down machine consists simply of weights, pulley, and a metallic cable rolled over pulleys. One end of the cable is attached to adjustable weights via a metallic rod and the other end is free to apply force by the user in order to lift the weights in upward direction. Pull-down machine is also known as "cable and pulleys" which is a multipurpose machine used to exercise for multi joints, biceps, triceps, shoulder, and legs. The working principle of the pull-down machine is mentioned in Fig 3, in which weights are selected by inserting a pin into specific cavity i.e., A, B, C, or D. If the pin entered into the cavity 'B', three upper most weights will be selected and lifted up for the purpose of exercise.

\subsection{Pull-down Machine as an Energy Generating Device}

The novelty of presented research is to explore the theoretical possibility to make "Pulldown" as an energy generating machine by

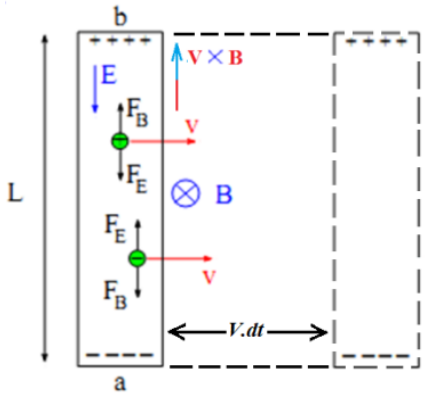

(a)

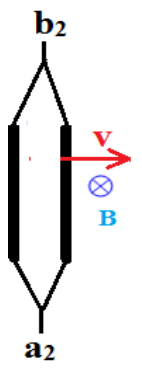

(b)

Figure 4. (a) Force on electrons under magnetic field in a conductor (b) Two conductor connected in parallel moving under the influence of magnetic field

modifying the structure of this machine. This machine can be made as an energy generating machine by installing a generator in it directly [16]. This research paper will propose a new 
way of alteration in order to convert a pull-down machine into an energy generating machine. According to the Faraday's law of electromagnetic induction, it states that "electro motive force (e.m.f)/ voltage is induced in a conductor when it is moved under the influence of magnetic field and cut the magnetic lines or whenever a conductor is placed under changing magnetic field (flux), the voltage is induced in it". The magnitude of induced voltage is directly proportional to the rate of change of magnetic flux. The mathematical form of Faraday's law for a conductor which is moving under the influence of magnetic field is:

$$
e_{\text {ind }}=\left|\frac{d \phi}{d t}\right|
$$

where $e_{\text {ind }}$ is induced voltage and $\frac{d \phi}{d t}$ is the rate of change of magnetic flux linkage. Voltage induced due to motion of conductor is known as motional e.m.f. Authors used the basic principles of physics \& electronics to prove mathematically that electrical energy can be generated from the weights of pull-down machine.

\subsection{Induced Voltage on a Conductor Moving in a Magnetic Field}

When a piece of wire with proper orientation is moved with velocity "V" through a magnetic field "B", according to Faraday's law of electro-magnetic induction, voltage will be induced in it and the amount of this voltage will be equal to the rate of change of magnetic flux. The expression for induced voltage in a piece of wire is given below:

$$
e_{\text {ind }}=(V \times B) \cdot L
$$

The angle between vectors $\mathrm{V} \& \mathrm{~B}$ is $90^{\circ}$ and the angle between " $\mathrm{L}$ " \& " $\mathrm{V} \times \mathrm{B}$ " is zero. The direction of resultant vector of cross product is upward, therefore the voltage on the conductor will be built up with positive at the top and negative at the bottom as represented in Fig 4(a).

$$
\begin{aligned}
& e_{\text {ind }}=(V B \sin \theta) L \cos \theta \\
& e_{\text {ind }}=(V B \sin 90) L \cos 0 \\
& e_{\text {ind }}=V B L
\end{aligned}
$$

The above expression of induced voltage can be derived by Faraday's law. Let the conductor travel a distance of " $V . d t$ " in a very short interval of time " $d t$ " and gain a new position which is shown by dotted lines in Fig 4(a). The magnetic flux " $d \phi$ " cut by conductor during time " $d t$ " will be equal to the product of area swept by the conductor and the magnetic flux density "B".

$d \phi=($ Area swept by conductor $) B$

$\Rightarrow d \phi=(L . V . d t) B$

The rate of cut/change of magnetic flux will become:

$$
\frac{d \phi}{d t}=V B L
$$

Conductor is moving perpendicular to the magnetic field, therefor magnetic force acting on a single negative charge (electron) will be in downward direction. Therefore, the charge will be separated in the conductor because the upper end will become positively charged and the lower end will become negatively charged, resulting in a potential difference. The 
" $\phi$ " is the magnetic field and " $\mathrm{B}$ " is the magnetic field density which is magnetic flux per unit area. Electric field lines travel from the positive end to the negative end are represented by " $\mathrm{E}$ ", the force on electron due to $\mathrm{E}$ is " $\mathrm{F}_{\mathrm{E}}$ ", " $\mathrm{q}$ " is the charge on electron and the magnetic force on electron is " $F_{B}$ ". Maximum potential difference occurs at the equilibrium state when both forces are equal. Conductor is moving perpendicular to the magnetic field so $\theta=90^{\circ}$ and forces on a charge particle are given below:

$$
\begin{gathered}
F_{B}=q V B \sin \theta=q V B \\
F_{E}=q E
\end{gathered}
$$

At equilibrium state, $F_{E}$ becomes equal to $F_{B}$ :

$$
E=V B
$$

Potential difference induced at end points will be:

$V_{a b}=V_{b}-V_{a}=E L$

$$
V_{a b}=V B L
$$

Above expression is the motional e.m. $f$, which will be induced in a single conductor. If there are 2 conductors connected in parallel as shown in Fig 4(b), the induced voltage at its ends " $a_{2}$ " \& " $\mathrm{b}_{2}$ " will also be " $V B L$ ". However current produced due to induced e.m.f will increase by two times. Similarly, for "N" conductors, the induced current will increase by "N" times, but the voltage will remain constant.

\subsection{Modification in Weights of Pull-down Machine}

Weights of the machine are supposed to be winded with a laminated copper wire. Winding will be made in such a way that the conductors of copper will remain perpendicular to the applied magnetic field. Winding on each weight will be in the form of

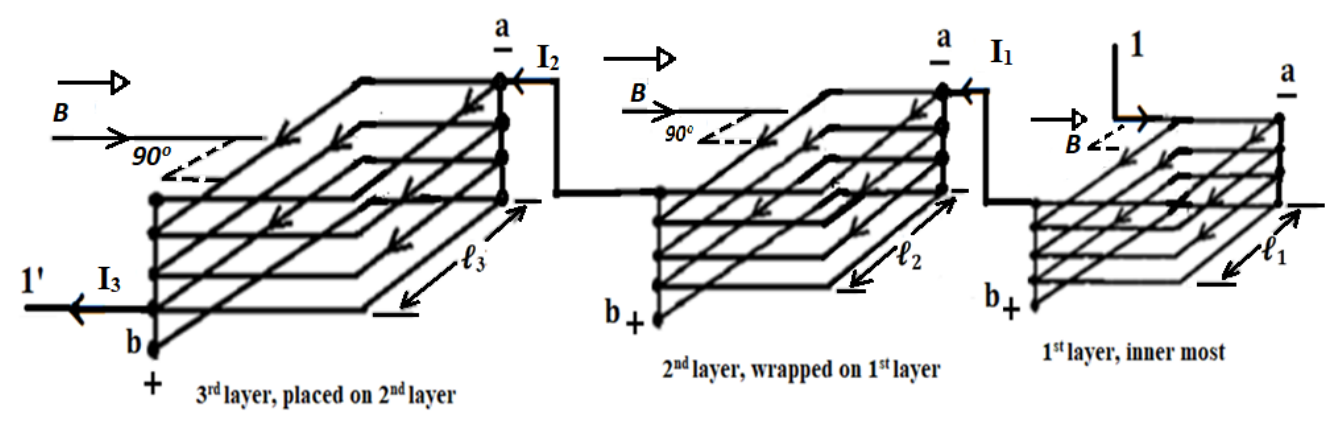

Figure 5. Induced current in 3 layers (parallel combination) windings on a single weight layers e.g., the $1^{\text {st }}$ layer of winding will be on the weight surface, when all surfaces of the weight are covered, and then a $2^{\text {nd }}$ layer will be made on the surface of the $1^{\text {st }}$ layer. After completion of the $2^{\text {nd }}$ layer, the $3^{\text {rd }}$ layer of turns will be wrapped on the second layer and so on. All three layers should be connected in series so that voltage induced by all layers 
will add up cumulatively. Suppose a single weight (upper most) says as " $w_{l}$ " having the inner most layer of conductors in which 4 conductors are on left and 4 conductors are on right side, so there are total of 8 conductors connected in parallel with each other. Let " $w_{1}$ " have 3 layers and every layer consists of 8 parallel connected conductors.

There will be different lengths of conductors for each layer such as $\boldsymbol{\ell}_{3}>\boldsymbol{\ell}_{2}>\boldsymbol{\ell}_{1}$ because these conductors are wrapped in the form of layers and all the layers are connected in series as shown in Fig 5. It is obvious that there are 8 numbers of conductors for each layer, however practically these numbers of conductors can vary for each layer. For the $1^{\text {st }}$ layer, 8 laminated conductors are connected in parallel circuit arrangement $\left(\mathrm{N}_{1}=8\right)$ therefor the total perpendicular length of the conductor will become $81_{1}$. Similarly if the $2^{\text {nd }}$ layer has $\mathrm{N}_{2}$ perpendicular conductors with total perpendicular length of $\mathrm{N}_{2} \mathrm{l}_{2}$ and the $3^{\text {rd }}$ layer will have total of $\mathrm{N}_{3} 1_{3}$ perpendicular length to the magnetic field. All conductors of each layer will be short circuited at point "a" \& "b" so that conductors will be in parallel circuit arrangement. In parallel circuit arrangement, there will be the same voltage on each conductor within a layer $e . g$, all conductors of the $1^{\text {st }}$ layer will have the same induced voltage however the current will be added up. Total induced current taken from 3 layers will be:

$$
I_{W 1}=I_{1}+I_{2}+I_{3}
$$

\subsection{Magnetic Field}

An electro magnet or a permanent magnet will be used for magnetic field. Electromagnet will be made by winding copper wire on a soft iron core and two ends of winding will be connected to a permanent D.C supply. Magnetic poles are placed around the weights of the machine as presented in Fig 6. Magnetic field lines travel from the North pole to the South pole, and they become perpendicular to the length of conductors which are present at the left and right sides of weights.

\subsection{Induced Voltage}

When these winded weights of the machine are lifted up by the user, the conductors which are perpendicular to the magnetic field will cut the magnetic field. According to Faraday's Law, electro-magnetic induction e.m.f

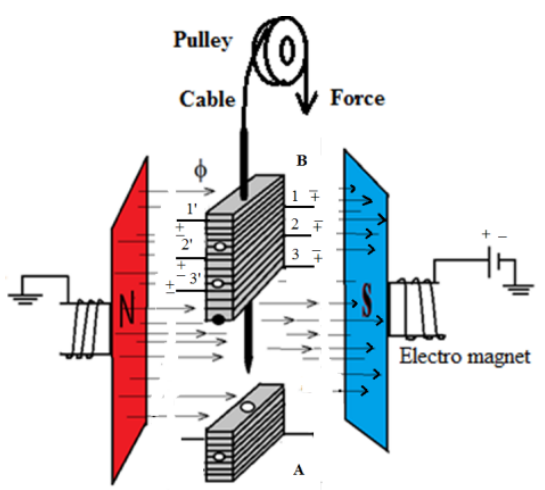

Figure 6. Pull-down machine as an energy generating machine having copper winding in on each weight will be induced in these conductors. Upward movement of weights is due to the external force applied by person and downward movement is due to gravity. As long as the exercise of lifting weights continues, the induction of e.m.f will also continue and reverse its polarity according to the direction of motion (upward or downward). Fig. 6 shows that 3 weights are selected to perform exercise. Two ends of winding of the upper most weight, the $2^{\text {nd }}$ weight, and the $3^{\text {rd }}$ weight are marked as $1 \& 1^{\prime}, 2 \& 2^{\prime}$; and $3 \& 3^{\prime}$, respectively. During upward motion of the weight, it is assumed that left ends of winding of all weights will become positive and right ends of windings will become negative. When weights move 
freely under the influence of gravity in downward direction, the polarity marks on the ends of windings will be reversed.

\subsection{Calculations of Induced e.m.f}

Let " $\phi$ " be the magnetic field and " $\mathrm{B}$ " is the magnetic field density which is magnetic flux per unit area $(\mathrm{B}=\phi / \mathrm{A}) . \mathrm{F}_{\text {ext }}$ is the external force which is applied by the user on the weights to lift them up with velocity " $\mathrm{V}$ " in upward direction. In order to calculate " $e_{l}$ " that is the induced voltage from $w_{l}$, it is necessary to add up induced voltages from all the layers of $w_{1}$. The motional e.m.f induced in the $1^{\text {st }}$ and $2^{\text {nd }}$ layers is given below:

$$
\begin{aligned}
& e_{L 1}=\mathrm{N}_{1} \ell_{1} V B \\
& e_{L 2}=\mathrm{N}_{2} \ell_{2} V B
\end{aligned}
$$

As it is mentioned in Fig 5 that all layers are connected in series therefor the induced voltage of all layers will be added up. Suppose that $w_{l}$ contains " $m$ " number of layers that are connected in series, and then the total induced voltage between the two ends $1 \& 1$ ' will be:

$$
e_{1}=\sum_{i=1}^{m}\left(N_{i} \cdot \ell_{i} \cdot B \cdot V\right)
$$

\subsection{Electrical Current}

Practically winding on weights has some resistance " $\mathrm{R}$ " in it. If " $\mathrm{I}_{\mathrm{w} 1}$ " is the total output current flowing from interval A to B (Fig 6) from " $w$ " and total induced motional e.m.f in $w_{1}$ is " $e_{1}$ " [17]:

$$
I_{w 1}=\frac{e_{1}}{R}
$$

\subsection{Mechanical Input Power}

When torque is applied on the pulley by external force, the pulley rotates. As applied force is increased torque in pulley will also increase. The amount of mechanical power supplied by the user is converted to the electrical form that is given by the following equation:

$$
P_{\text {in }}=\text { Force } \times \text { Velocity }=F V
$$

With respect to angular velocity of pulley, the power supplied is

$P_{\text {in }}=($ Torque in pulley $)($ Angular velocity of pulley $)$

$$
P_{\text {in }}=\tau . \omega
$$

By comparing equation (3.12) \& (3.13) gives rise to: 


$$
F . V=\tau . \omega
$$

If " $r$ " is the radius of pulley, equation (3.14) is changed by multiplying " $r$ " on both sides:

$$
r(F . V)=\tau . r \omega
$$

In circular motion, the relation between linear and angular velocity is $\mathrm{V}=\mathrm{r} . \omega$, so equation (3.15) becomes:

$$
r . F=\tau
$$

This is the amount of torque which induces e.m.f in a pull-down machine.

\subsection{Electrical output power}

If " $R$ " is the resistance of winding of selected weight, the output electrical power from a single weight (say $\mathrm{w}_{1}$ ) will be:

$$
P_{\text {out }}=e_{t} I_{w 1}-I_{w 1}{ }^{2} R
$$

Electrical output power will always be less than the mechanical input power due to friction, heat, and electrical losses.

\section{Scope of EGGS}

The concept of energy generating gymnasium system (EGGS) has a great scope in developing countries where youths are becoming more fitness conscious [18]. Solar and wind power generation are the most famous renewable energy resource among the energy consumers. In this research, authors are describing another energy generating source which is definitely renewable, and this energy source is human itself [14]. The fundamental idea of EGGS is shown in Fig 7, in this system the energy generating gymnasium machines (EGGMs) are figured as $\mathrm{M}_{1}$ to $\mathrm{M}_{4}$. These EGGMs supply power to A.C and D.C appliances within the gymnasium building. EGGS will be very beneficial for those people who are running the gymnasium business. The gymnasium owners could be able to cut down the electricity expenses and get free energy (electrical energy) from the users who come in gymnasium for exercise. So, the owners would be able to get double benefits. One is receiving money in the form of fee and second one is to enjoy free energy. Normally, a gymnasium runs 16 hours per day. However, for a crowded area, a gymnasium may be opened for 24 hours a day. So, the output of all the gymnasium machines will give a bulk amount of electrical energy. The excessive electrical energy can be sold back to the utility [19]. Smart grid system which is evolving for the power delivery in the $21^{\text {st }}$ century enables the customer to sell excess of electric energy from distributed energy sources (DES) back to the utility via micro grid (MG). MG is a small grouping of interconnected power generation and control technologies, which can operate within or independent of a central grid and increasing system reliability. By enabling the integration of distributed generation (DG) such as wind and solar, these systems can be more flexible than traditional grids. 
EGGS will be taken as a new distributed power generating source in the field of renewable energy resources.

\section{Conclusions}

In the proposed theoretical model, it is concluded that the expended energy by people during exercise in gymnasium in order to burn their calories to achieve their desired fitness level can be converted and stored in batteries in the form of electrical energy. EGGS are very beneficial for the gymnasium owners, because this system would make the gymnasium independent in the energy production. The energy harvested from EGGM will be green, clean, eco-friendly, and renewable. Distributed EGGS in all over the world will definitely play an important role in business and pay its share in renewable and sustainable energy resources.

\section{Summary}

The main idea behind the research is to make electricity from men power and then store this electrical energy in D.C batteries. Presented research theoretically suggests that the energy spent in gymnasium should be converted into electrical energy, for this purpose gymnasium machines are modified into EGGM by using copper windings and

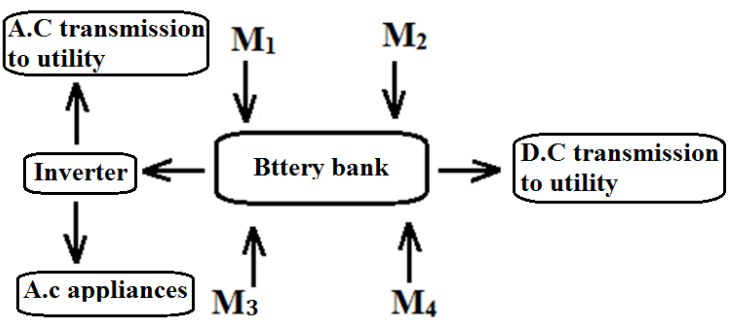
magnets. Whenever these machines are operated, the human exerted energy is then utilized to charge batteries. Excess of energy could be sold back to the national

Figure 7. Block diagram of EGGS utility through MG. Man takes his energy from food which is stored in human body in the form of ATP. During different types of exercises ATP-ADP conversion is also presented.

\section{Acknowledgement}

Authors would like to express the sincere and special thanks to their colleagues for permitting them in gymnasium to make observation and analysis of different available machines and equipment.

\section{CONFLICTS OF INTEREST}

The authors declare that there is no conflict of interests regarding the publication of this paper. 


\section{REFERENCES}

[1] International Energy Agency. (2017). Key world energy statistics. https://www.iea.org/publications/freepublications/publication/KeyWorld2017.pdf (last accessed on October 7, 2018).

[2] U.S. Energy Information Administration (EIA). (2017). International Energy Outlook 2017. https://www.eia.gov/outlooks/ieo/pdf/0484(2017).pdf (last accessed on October 7, 2018).

[3] The Great Outdoor Gym Company. (2018). Green Energy. http://www.tgogc.com/Green-Energy.html (last accessed on October 7, 2018)

[4] The Great Outdoor Gym Company. (2018). Lumi Plus. http://www.tgogc.com/Products/TGO-Energy-Play...-Coming-Soon/LumiPlus.Html?cPath=7 175 (last accessed on October 7, 2018).

[5] Strzelecki, R., Jarnut, M., and Benysek, G. (2007). Exercise bike powered electric generator for fitness club appliances. In: Proc., Power Electronics and Applications, 2007 European Conference on, IEEE, pp: 1-8.

[6] World Energy Council. (2016). World Energy Resources 2016. https://www.worldenergy.org/wp-content/uploads/2016/10/World-Energy-

Resources-Full-report-2016.10.03.pdf (last accessed on October 7, 2018).

[7] Verma, P. S., V. K. Agarwal, and P. S. Verma. (2007). Cell biology, genetics, molecular biology, evolution and ecology. S. chand \& Company Limited.

[8] Herman, I.P. (2016). Physics of the Human body. Springer.

[9] Manoj, K.M. (2007). Mitochondrial oxidative phosphorylation: Debunking the concepts of electron transport chain, proton pumps, chemiosmosis and rotary ATP synthesis, arxiv preprint arxiv: 1703.05826.

[10] DuPlesis, S.S, Agarwal, A. Mohanty, G., and Vander Lind, M. (2015). Oxidative phosphorylation versus glycolysis: What fuel do spermatozoa use? Asian journal of andrology, 17(2), 230.

[11] Martin, W.F, Thauer, R.K. (2017). Energy in ancient metabolism. Cell, 168(6), 953-955.

[12] Lodish, H. Berk, A. Matsudaria, P. Kaiser, C.A, Krieger, M. Scott, M.P, Zipursky, S.L, Darnell. (2004) Molecular cell biology $\left(5^{\text {th }}\right.$ ed). New York.NY:W.H. Freeman.

[13] Gustin, P.B. (2001). Energy system interaction and relative contribution during maximal exercise. Sports medicine, 31(10), 725-741.

[14] Bidwai, M.S., Jaykar, M.A. and Shinde, M.S. (2017). Gym Power Station: Turning Workout into Electricity. International Research Journal of Engineering and Technology (IRJET). 4(03), 424-426.

[15] Borchate, S., Gaikwad, A., Jadhav, A., Dhage, P. (2017) Design of Treadmill to Generate Electricity by using Mechanical Energy. International Conference on Ideas, Impact and Innovation in Mechanical Engineering (ICIIIME 2017) 5 (04), 498-505.

[16] Bonde, V.S., Khatake, B.V., Zambare, D.V., Patel, V.D., Kadam, N.V. (2018). Electric Power Generation from Gym Equipment with Polarity Checker and Changer Circuit. International Journal for Scientific Research \& Development, 5(02), 992-995.

[17] Chapman, S.J. (2005). Electric Machinery Fundamentals $\left(4^{\text {th }} \cdot \mathrm{ed}\right)$. New York, NY: McGraw-hill. 
[18] Gaurav, H., Nikhurpa, K.S., Chaudhary, D., Feroz, W. (2015). Energy harvesting through smart gym. In Proceedings of National Conference on "Emerging Trends in Electronics \& Communication” (ETEC-2015), 1(02), 37-42.

[19] Kumar, M., Mundada, G.S. (2017). Energy Harvesting from Gym Equipments. International Journal of Innovative Research in Electrical, Electronics, Instrumentation and Control Engineering. 5 (07), 127-131. DOI 10.17148/IJIREEICE.2017.5721

Article copyright: (C) 2019 M. Musharraf, Ifrah Saleem, and Dr. Farhat Iqbal. This is an open access article distributed under the terms of the Creative Commons Attribution 4.0 International License, which permits unrestricted use and distribution provided the original author and source are credited. 